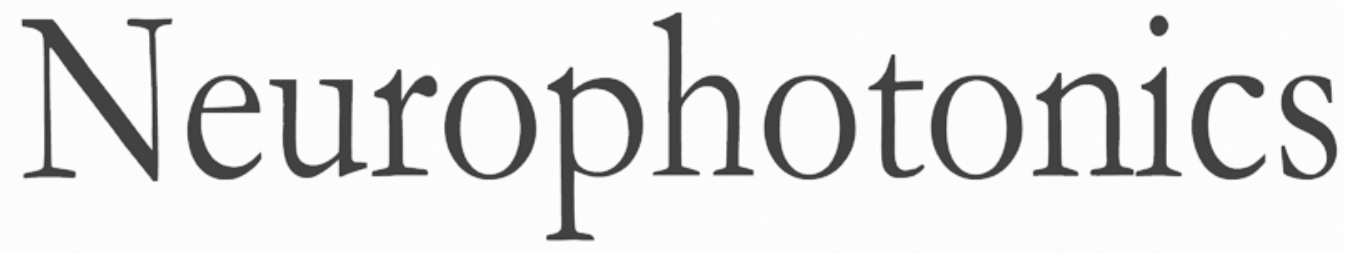

\title{
Prolonged monitoring of cerebral blood flow and autoregulation with diffuse correlation spectroscopy in neurocritical care patients
}

Juliette Selb

Kuan-Cheng Wu

Jason Sutin

Pei-Yi (Ivy) Lin

Parisa Farzam

Sophia Bechek

Apeksha Shenoy

Aman B. Patel

David A. Boas

Maria Angela Franceschini

Eric S. Rosenthal 


\title{
Prolonged monitoring of cerebral blood flow and autoregulation with diffuse correlation spectroscopy in neurocritical care patients
}

\author{
Juliette Selb, ${ }^{a}$ Kuan-Cheng Wu, ${ }^{a}$ Jason Sutin, ${ }^{a}$ Pei-Yi (Ivy) Lin, ${ }^{a}$ Parisa Farzam, ${ }^{\text {a }}$ Sophia Bechek, ${ }^{b}$ \\ Apeksha Shenoy, ${ }^{b}$ Aman B. Patel, ${ }^{b}$ David A. Boas, ${ }^{a}$ Maria Angela Franceschini, ${ }^{a, t, *}$ and Eric S. Rosenthal ${ }^{b, \dagger}$ \\ aMassachusetts General Hospital, Optics at Martinos, Athinoula A. Martinos Center for Biomedical Imaging, Department of Radiology, \\ Charlestown, Massachusetts, United States \\ ${ }^{\mathrm{b}}$ Massachusetts General Hospital, Department of Neurology, Boston, Massachusetts, United States
}

\begin{abstract}
Monitoring of cerebral blood flow (CBF) and autoregulation are essential components of neurocritical care, but continuous noninvasive methods for CBF monitoring are lacking. Diffuse correlation spectroscopy $(\mathrm{DCS})$ is a noninvasive diffuse optical modality that measures a CBF index $\left(\mathrm{CBF}_{\mathrm{i}}\right)$ in the cortex microvasculature by monitoring the rapid fluctuations of near-infrared light diffusing through moving red blood cells. We tested the feasibility of monitoring $\mathrm{CBF}_{\mathrm{i}}$ with $\mathrm{DCS}$ in at-risk patients in the Neurosciences Intensive Care Unit. DCS data were acquired continuously for up to $20 \mathrm{~h}$ in six patients with aneurysmal subarachnoid hemorrhage, as permitted by clinical care. Mean arterial blood pressure was recorded synchronously, allowing us to derive autoregulation curves and to compute an autoregulation index. The autoregulation curves suggest disrupted cerebral autoregulation in most patients, with the severity of disruption and the limits of preserved autoregulation varying between subjects. Our findings suggest the potential of the DCS modality for noninvasive, long-term monitoring of cerebral perfusion, and autoregulation. () 2018 Society of Photo-Optical Instrumentation Engineers (SPIE) [DOI: 10.1117/1.NPh.5.4.045005]
\end{abstract}

Keywords: diffuse correlation spectroscopy; near-infrared spectroscopy; cerebral blood flow; cerebral autoregulation; neuromonitoring; subarachnoid hemorrhage; neurocritical care.

Paper 17131RR received Nov. 15, 2017; accepted for publication Sep. 24, 2018; published online Nov. 13, 2018; corrected Feb. 11, 2020.

\section{Introduction}

Cerebral autoregulation (CA) is a protective mechanism of the healthy brain vasculature that maintains relatively constant cerebral perfusion over a broad range of cerebral perfusion pressure (CPP). ${ }^{1} \mathrm{CA}$ is commonly disrupted following brain injuries, such as stroke, subarachnoid hemorrhage $(\mathrm{SAH})$, or traumatic brain injury. ${ }^{1-7}$ This impairment complicates the management of brain injured patients, as it can lead to secondary brain insult through ischemic or hyperperfusion mechanisms. Monitoring of cerebral blood flow (CBF) and assessment of CA are, therefore, essential components of neurocritical care. ${ }^{5,8}$

Traditional modalities for imaging cerebral perfusion, including $\mathrm{PET}^{9}$ and MRI, ${ }^{10}$ only provide a snapshot of the brain status and not continuous monitoring. Continuous measures of cerebral perfusion afforded by laser Doppler flowmetry, ${ }^{11}$ thermal diffusion, ${ }^{12}$ focal brain tissue oxygen tension, ${ }^{13}$ or intracranial pressure (ICP) as a surrogate for cerebral blood volume (CBV) are all invasive and offer only a focal or global measure. ${ }^{14,15}$ These continuous monitoring modalities have limited clinical practicality in mild and moderate brain injuries, as they require the placement of invasive probes. Additionally, their invasive nature does not afford the possibility of multiregional assessment.

Transcranial Doppler (TCD) ultrasonography ${ }^{16,17}$ measures the $\mathrm{CBF}$ velocity $(\mathrm{CBFv})$ in the main arteries supplying the

\footnotetext{
*Address all correspondence to: Maria Angela Franceschini, E-mail: mari@ nmr mgh.harvard.edu

†Senior authors contributed equally to this manuscript
}

brain. Due to its noninvasiveness, low-cost, and high temporal resolution, the technique has become a tool of choice for assessing CA in clinical settings. ${ }^{17}$ However, it is not always possible to obtain a good TCD signal through the cranial windows, and the technology is not adapted to long recording periods because the transducers can move easily or require cumbersome gear. ${ }^{17}$ Finally, the upstream measure of CBFv in major arteries may not reflect regional perfusion in the smaller cerebral vasculature.

Cerebral oximeters based on near-infrared spectroscopy (NIRS) retrieve relative cerebral oxygen saturation $\mathrm{rSO}_{2}$, are less cumbersome than TCD, and allow noninvasive continuous monitoring over long recording times. $\mathrm{rSO}_{2}$ has been proposed as a surrogate for local $\mathrm{CBF}$, and cerebral oximeters have the potential to assess CA continuously in clinical settings. ${ }^{18-21}$ However, the use of $\mathrm{rSO}_{2}$ as a surrogate for $\mathrm{CBF}$ relies on many assumptions, ${ }^{22}$ such as constant hematocrit, arterial saturation, and cerebral oxygen metabolism, which may not hold true especially in pathological and surgical conditions. Research about NIRS devices that allow measurement of both oxy- and deoxy-hemoglobin changes as an indirect metric for cerebral perfusion has also been proposed to assess CA. ${ }^{23-27}$ But in addition to the indirect measure of perfusion, NIRS in adults generally suffers from the contamination of the signal by extracerebral vasculature reflecting systemic hemodynamics. ${ }^{28-31}$

In the present study, we tested the feasibility of applying diffuse correlation spectroscopy (DCS) to monitoring CBF and CA in neurocritical care patients. Like NIRS, DCS uses nearinfrared light propagating diffusely through the head, but its

2329-423X/2018/\$25.00 (C) 2018 SPIE 
contrast arises from the motion of the light-scattering red blood cells, and it retrieves a blood flow index $\left(\mathrm{BF}_{\mathrm{i}}\right)$ that has been demonstrated to correlate well with gold standard measures of $\mathrm{CBF}^{32-35}$ DCS has the NIRS benefits of nonionizing radiation, noninvasiveness, high temporal resolution (albeit generally lower than NIRS), and high portability and flexibility. In addition to the fact that it provides a direct estimate of CBF, it has been shown to have better brain sensitivity than NIRS. ${ }^{36}$ Therefore, the DCS modality has promising characteristics to overcome some of the limitations of existing clinical modalities described above.

First proposed in $1997,{ }^{37}$ the technique has seen recent advances in terms of instrumentation, ${ }^{38,39}$ theoretical modeling of the signal, ${ }^{40,41}$ and data analysis, ${ }^{42-45}$ leading to a growing number of applications for human and clinical research. ${ }^{46-59}$ Cheng et al. ${ }^{55}$ first proposed to monitor CA with DCS in healthy subjects, by measuring the phase shift between arterial blood pressure and $\mathrm{BF}_{\mathrm{i}}$ low-frequency oscillations (LFOs). They found the method to be most robust when enhancing LFOs through head-of-bed tilt or enforced breathing at $0.1 \mathrm{~Hz}$. In the present work, the critical condition of the studied population precluded such physiological manipulations.

Independently of the modality to assess CBF directly or indirectly, note that the complete autoregulation curve, from below the lower limit to beyond the upper limit of CA, or Lassen curve, ${ }^{60}$ is rarely observed clinically because CPP generally does not reach these extreme values spontaneously, and inducing them through maneuvers or pharmaceutical interventions can be unsafe. Instead, clinical monitoring often relies on assessing the autoregulatory response to spontaneous low-frequency fluctuations in blood pressure. Continuous assessment of CA can be derived from "reactivity indices," computed as moving correlation coefficients between CBF surrogate and the slow waves of CPP, or alternatively mean arterial pressure (MAP). ${ }^{15}$ Different indices have been termed Mx, Mxa, PRx, PAx, TOx, etc. depending on the pressure parameter (CPP or MAP) and the $\mathrm{CBF}$ surrogate parameter $\left(\mathrm{CBFv}, \mathrm{ICP}, \mathrm{rSO}_{2}\right.$, etc.) used in its computation. In general, impaired autoregulation is characterized by CBF passively following CPP fluctuations, resulting in high reactivity indices $(>0.2$ to 0.4$)$, ${ }^{15}$ whereas preserved autoregulation is characterized by low reactivity indices.

Here, we assess the feasibility of DCS to monitor CBF continuously over long periods of time in the challenging environment of the Neurosciences Intensive Care Unit (NeuroICU). We also investigate the possibility to assess $\mathrm{CA}$ in response to spontaneous blood pressure fluctuations in neurocritical care patients.

\section{Materials and Methods}

\subsection{Study Design and Patients}

We recruited six patients (two males, four females; age range 57 to 66 years old, median 64) admitted to the Massachusetts General Hospital NeuroICU for aneurysmal SAH. Patients were enrolled under two protocols approved by the MGH Institutional Review Board. The patient's legally authorized representative provided written informed consent before any procedure related to the research study started.

The optical probe was positioned and attached on the patient's scalp so as not to interfere with clinical monitoring equipment, and DCS data were recorded continuously, and

Table 1 Clinical and study characteristics of all patients. Sex: M, male; F, female; SAH grade at admission; HH, Hunt and Hess grade; F, Fisher grade; ruptured aneurysm site; MCA, middle cerebral artery; PComm, posterior communicating artery; optical monitoring; SD, source-detector separation.

\begin{tabular}{|c|c|c|c|c|c|c|c|c|c|c|c|c|}
\hline \multicolumn{6}{|c|}{ Patient info } & \multicolumn{7}{|c|}{ Optical monitoring (DCS) } \\
\hline \multirow[b]{2}{*}{$\#$} & \multirow[b]{2}{*}{ Age } & \multirow[b]{2}{*}{ Sex } & \multirow[b]{2}{*}{$\begin{array}{c}\text { SAH } \\
\text { grade at } \\
\text { admission }\end{array}$} & \multirow[b]{2}{*}{$\begin{array}{l}\text { Ruptured } \\
\text { aneurysm } \\
\text { site }\end{array}$} & \multirow[b]{2}{*}{ Outcome } & \multirow[b]{2}{*}{$\begin{array}{l}\text { Days after } \\
\text { admission }\end{array}$} & \multicolumn{3}{|c|}{ Recording duration } & \multirow[b]{2}{*}{$\begin{array}{l}\text { Probe } \\
\text { location }\end{array}$} & \multirow[b]{2}{*}{$\begin{array}{c}\text { Short } \\
\text { SD } \\
(\mathrm{mm})\end{array}$} & \multirow[b]{2}{*}{$\begin{array}{l}\text { Long } \\
\text { SD } \\
(\mathrm{mm})\end{array}$} \\
\hline & & & & & & & $\begin{array}{l}\text { Total } \\
\text { (min) }\end{array}$ & $\begin{array}{l}\text { Kept } \\
\text { (min) }\end{array}$ & $\begin{array}{c}\text { Kept } \\
(\% \text { total })\end{array}$ & & & \\
\hline \multirow[t]{2}{*}{1} & 57 & M & $\mathrm{HH} 3, \mathrm{~F} 3$ & Left ICA & $\begin{array}{l}\text { Discharged } \\
\text { at } 30 \text { days }\end{array}$ & 21 & 116 & 78 & $67 \%$ & \multirow[t]{2}{*}{$\begin{array}{l}\text { Right } \\
\text { frontal }\end{array}$} & 8 & 28 \\
\hline & & & & & & 22 & 133 & 131 & $99 \%$ & & 8 & 28 \\
\hline \multirow[t]{2}{*}{2} & 63 & $\mathrm{~F}$ & $\mathrm{HH} 4, \mathrm{~F} 3$ & $\begin{array}{l}\text { Right, } \\
\text { PComm }\end{array}$ & $\begin{array}{l}\text { Discharged } \\
\text { at } 23 \text { days }\end{array}$ & 3 & 500 & 448 & $90 \%$ & \multirow[t]{2}{*}{ Left frontal } & 9 & 26 \\
\hline & & & & & & 6 & 1227 & 987 & $80 \%$ & & 9 & 26 \\
\hline \multirow[t]{2}{*}{3} & 66 & $\mathrm{~F}$ & $\mathrm{HH} 4, \mathrm{~F} 3$ & $\begin{array}{l}\text { Right MCA, } \\
\text { Left MCA, }\end{array}$ & Deceased & 2 & 807 & 738 & $91 \%$ & \multirow[t]{2}{*}{$\begin{array}{l}\text { Right } \\
\text { frontal }\end{array}$} & 8 & 20 \\
\hline & & & & Left PComm & & 3 & 633 & 610 & $96 \%$ & & 8 & 23 \\
\hline 4 & 58 & $\mathrm{~F}$ & $\mathrm{HH} 4, \mathrm{~F} 4$ & Right MCA & $\begin{array}{l}\text { Discharged } \\
\text { at } 17 \text { days }\end{array}$ & 8 & 990 & 220 & $22 \%$ & $\begin{array}{l}\text { Right } \\
\text { frontal }\end{array}$ & 9 & 22 \\
\hline \multirow[t]{2}{*}{5} & 65 & M & $\mathrm{HH} 4, \mathrm{~F} 3$ & Right A1 & Deceased & 3 & 755 & 755 & $100 \%$ & \multirow[t]{2}{*}{ Left frontal } & 8 & 20 \\
\hline & & & & & & 7 & 380 & 380 & $100 \%$ & & 8 & 21 \\
\hline \multirow[t]{2}{*}{6} & 66 & $F$ & $\mathrm{HH} 5, \mathrm{~F} 4$ & Right MCA & $\begin{array}{l}\text { Discharged } \\
\text { at } 26 \text { days }\end{array}$ & 3 & 180 & 96 & $53 \%$ & \multirow[t]{2}{*}{$\begin{array}{l}\text { Right } \\
\text { frontal }\end{array}$} & 5 & 20 \\
\hline & & & & & & 5 & 240 & 240 & $100 \%$ & & 5 & 20 \\
\hline
\end{tabular}


synchronously to clinically monitor physiological signals. The timing of DCS data acquisition for the patient cohort ranged from postadmission day 2 to 22. Patients were studied on one to three separate days, with recording sessions lasting between $10 \mathrm{~min}$ and $20.5 \mathrm{~h}$, as permitted by clinical care. Only data from recording sessions longer than $60 \mathrm{~min}$ were included in the analysis and are reported here.

The clinical and study characteristics of all patients are shown in Table 1.

\subsection{Diffuse Correlation Spectroscopy Measurements}

\subsubsection{Diffuse correlation spectroscopy device}

Optical data were acquired using a custom-built DCS system, similar to that originally developed by Durduran et al., ${ }^{61}$ based on a long coherence-length laser (CrystaLaser, Reno, Nevada) emitting at $785 \mathrm{~nm}$, and four photon-counting avalanche photodiodes (Perkin-Elmer, Québec, Canada) whose counting signals are converted to temporal intensity autocorrelation curves using an eight-channel correlator, or a custom-built field-programmable gate array (FPGA)-based correlator board ${ }^{62}$ in one patient. DCS data were acquired at $1 \mathrm{~Hz}$.

\subsubsection{Diffuse correlation spectroscopy probes}

We designed and built multidistance optical probes targeted to clinical applications. Specifically, we used two types of probes, as shown in Fig. 1: an early version, relatively heavy and rigid, with DCS fibers at two source-detector separations [Fig. 1(a)]; a second, lighter, and more flexible version for DCS-only measurements at up to four separations, three-dimensional printed in a rubber-like material [Fig. 1(b)]. The probes contain the fiber ends and small prisms to provide the contact between the fiber ends and the scalp. Illumination is performed with a $200-\mu \mathrm{m}$ multimode fiber. A small diffuser is placed between the illumination fiber end and the prism to enlarge the illumination beam [Fig. 1(c)], and enables using higher incident power while remaining within the ANSI standards for safe skin exposure. ${ }^{64}$ For the detection, we use single-mode $5-\mu \mathrm{m}$ diameter fibers. Three fibers were located at the long separation, and the signals from the three detectors were averaged. The short source-detector separation varied between 5 and $9 \mathrm{~mm}$, and the long separation between 20 and $28 \mathrm{~mm}$, depending on DCS signal quality and space availability. The exact values for each recording are shown in Table 1.

\subsubsection{Probe placement}

For each patient, the DCS probe was placed on the scalp where permitted by other clinical neuromonitoring gear and where the DCS signal was deemed satisfactory by the research staff (photon count over 10,000 per second, autocorrelation curves visually indicative of a physiological signal, with a coherence factor $\beta$ of at least 0.3 , and the tail of the curve reaching $\sim 1$ ). The location was always over fronto-temporal regions, either on the hemisphere ipsilateral or contralateral to any parenchymal hemorrhage. The probe was attached with gauze and a clinical adhesive (collodion). Figures 1(d) and 1(e) show pictures of the first and second probes, respectively, attached on a patient scalp. The photographs also show scalp EEG electrodes, stitches from craniotomy surgery, and an intraventricular ICP catheter.

\subsection{Auxiliary Recordings}

Simultaneously to the DCS data, we recorded the patient's physiological signals that were part of their clinical monitoring. In relevance to the present study, these included invasive mean arterial blood pressure (MAP), ICP, CPP, and white matter perfusion from a thermal diffusion invasive probe (Hemedex Inc., Cambridge, Massachusetts) in four patients. However, the recording of white matter perfusion had to be discarded in
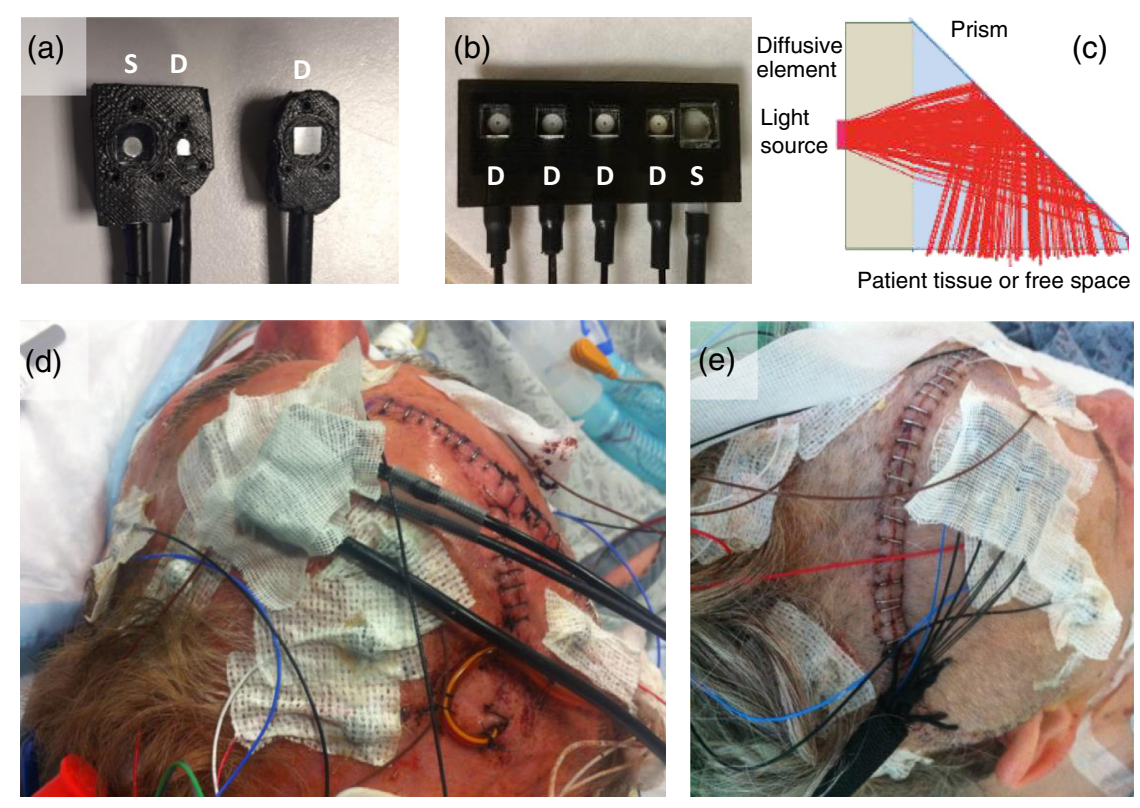

Fig. 1 DCS probes. (a) First probe version. The probe is made of two parts so that the separation can be optimized based on the signal quality (b). Second DCS-only probe version, with multiple fibers at each detector location. (c) Schematic of the illumination prism with a diffuser enlarging the beam diameter $\left(\right.$ patent ${ }^{63}$ ). (d) First probe placed on a patient and attached with gauze and collodion. (e) Second DCS-only probe attached on a patient. (S, source; D, detector). 
two of these patients due to low-signal quality of the measurements. To align the time series of the DCS data and of the physiological data in postprocessing, we sent a transistortransistor logic pulse train of varying frequencies to the DCS device and to the clinical neuromonitor (Moberg CNS). As proposed in the literature, we computed the cerebrovascular pressure reactivity index $\mathrm{PRx}^{15}$ as the moving Pearson correlation coefficient, over a 5-min window, between 10-s time-averaged values of ICP and MAP.

\subsection{Data Processing}

In brief, we compute a CBF index at each time point, as well as an autoregulation index BFAx. Then, we derive two curves of autoregulation (flow versus pressure and autoregulation index versus pressure) for each recording. The steps are detailed below.

\subsubsection{Diffuse correlation spectroscopy data analysis}

The autocorrelation curves were averaged using a 10-s moving window to improve the SNR at each time point. Then, the averaged autocorrelation curve at each time point and for each source-detector separation was fitted with the analytical solution of the correlation diffusion equation for a semi-infinite homogenous medium, ${ }^{37}$ assuming constant optical properties of absorption $\mu_{a}=0.15 \mathrm{~cm}^{-1}$ and reduced scattering $\mu_{s}^{\prime}=10 \mathrm{~cm}^{-1}$ to retrieve a $\mathrm{BF}_{\mathrm{i}}$ at each time point and each separation. All data $\left(\mathrm{BF}_{\mathrm{i}}\right.$ and auxiliary clinical recordings) were aligned on the same time scale and resampled with a 1-s step. The data were then visually inspected, and segments with strong motion artifacts were manually identified and discarded from further analysis. Relying on the assumption that the measured $\mathrm{BFi}$ at the long separation $\mathrm{BFi}_{\text {Long }}$ should be higher than that at the short separation $\mathrm{BFi}_{\text {Short }}$ because its sensitivity to the brain is higher and because CBF should be higher than scalp flow, ${ }^{65}$ in postanalysis we discarded autoregulation curves for which $\mathrm{BFi}_{\text {Long }}<\mathrm{BFi}_{\text {Short }}$. Finally, on the remaining data, a cerebral blood flow index $\left(\mathrm{CBF}_{\mathrm{i}}\right)$ was assessed by subtracting a fixed contribution $(70 \%)$ of the $\mathrm{BF}_{\mathrm{i}}$ at the short source-detector separation from the $\mathrm{BF}_{\mathrm{i}}$ at the long source-detector separation $\mathrm{CBF}_{\mathrm{i}}=\mathrm{BFi}_{\text {Long }}-0.7 \times \mathrm{BFi}_{\text {Short }}$. Because the estimation of this contribution is arbitrary and bound to vary across subjects, we also compared the results when using simply $\mathrm{BFi}_{\text {Long }}$ instead of $\mathrm{CBF}_{\mathrm{i}}$ to verify that the trends remain similar. Finally, the $\mathrm{CBF}_{\mathrm{i}}$ values were normalized by the median over the whole recording, resulting in a time-dynamic relative cerebral blood flow index $\mathrm{rCBF}_{\mathrm{i}}$.

\subsubsection{Autoregulation index}

We computed an autoregulation index, similar to those proposed in the literature: ${ }^{15}$ first, MAP was smoothed using a 10 -s moving window, then we computed the running Pearson correlation coefficient between $\mathrm{rCBF}_{\mathrm{i}}$ and MAP over a moving 5-min window. We designated this blood flow autoregulation index BFAx to distinguish it from other similar indices described in the literature.

\subsubsection{Autoregulation curves}

To estimate the autoregulation curves of a patient over the course of each recording, MAP values were divided into groups of $5 \mathrm{mmHg}$ (e.g., $80 \leq \mathrm{MAP}<85 \mathrm{mmHg}$ ) and $\mathrm{rCBF}_{\mathrm{i}}$ was averaged within each group. Using the same approach, the autoregulatory index BFAx was binned in groups of 5-mmHg width of the CPP values to obtain an averaged curve of BFAx versus CPP for each recording. The BFAx versus CPP curves were qualitatively compared with those of PRx versus CPP obtained in the same manner.

\subsubsection{Comparison of $\mathrm{rCBF}_{i}$ and invasive measure of white matter perfusion}

In the two patients for whom an invasive measure of white matter perfusion was available and displayed sufficient quality, we compared it with the DCS measure of $\mathrm{rCBF}_{\mathrm{i}}$ by computing the correlation coefficient between the two metrics over the duration of the recording. Segments of recordings during which the Hemedex probe is recalibrating were excluded from this analysis.

\section{Results}

\subsection{Signal Quality}

The DCS monitoring duration for each recording varied between 2 and $20 \mathrm{~h}$ (mean $9 \mathrm{~h}$ ), with mean of $7 \mathrm{~h}$ of data kept in subsequent analysis for each recording. An average of $18 \%$ of the data was discarded because of motion artifacts, or in one case because of the probe getting detached.

\subsection{Correlation Between $\mathrm{rCBF}_{i}$ and Invasive Perfusion Measure}

White matter perfusion $\mathrm{BF}_{\mathrm{WM}}$ was measured by an invasive diffusion probe with sufficient data quality in two patients. Figure 2 shows the scatter plots of $\mathrm{rCBF}_{\mathrm{i}}$ versus $\mathrm{BF}_{\mathrm{WM}}$ for the two patients. In patient 3 , the $\mathrm{BF}_{\mathrm{WM}}$ measured by the invasive diffusion probe showed good correlation (correlation coefficient $R=0.59$ ) with the DCS-derived $\mathrm{rCBF}_{\mathrm{i}}$. Instead, in patient 2, there was no correlation between $\mathrm{BF}_{\mathrm{WM}}$ and $\mathrm{rCBF}_{\mathrm{i}}(R=-0.03)$. Note, however, that when selecting shorter segments of data in this patient, the correlation between the two signals increased significantly. For instance, when considering only the last $4 \mathrm{~h}$ of recording (as opposed to the total $16 \mathrm{~h}$ ), $\mathrm{BF}_{\mathrm{WM}}$ and $\mathrm{rCBF}_{\mathrm{i}}$ were significantly correlated with a correlation coefficient of $R=0.62$.
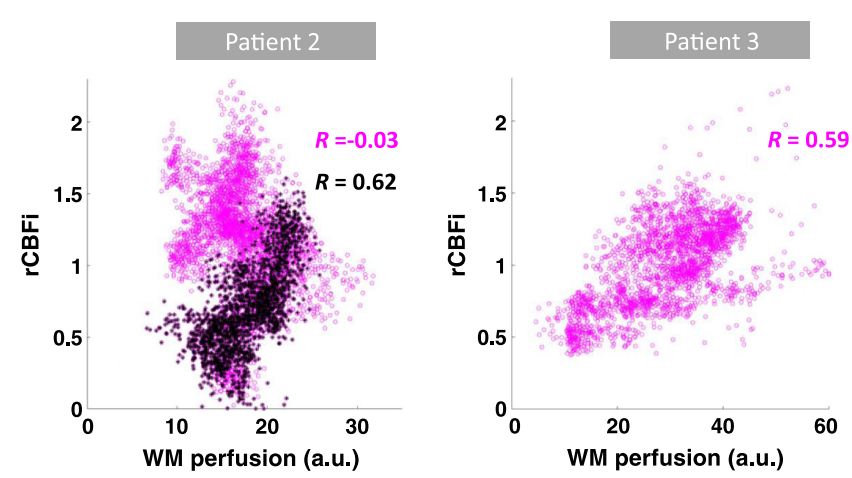

Fig. 2 Scatter plots of the DCS-derived relative CBF index versus white matter perfusion measured with the invasive diffusion sensor in two patients. In patient 2, the data points corresponding to the last $4 \mathrm{~h}$ of recording are plotted in black. 


\subsection{Autoregulation Curves}

The results of CBFi measurements and of autoregulation indices BFAx and PRx for all subjects are shown in Figs. 3-8. For each figure, multiple rows correspond to different recordings. The first column displays the simultaneous $\mathrm{rCBF}_{\mathrm{i}}, \mathrm{ICP}$, and CPP time traces over up to $6 \mathrm{~h}$. The second column displays the $\mathrm{rCBF}_{\mathrm{i}}$ versus MAP curves for that recording (i.e., parts of the static autoregulation curve). The third and fourth columns present the evolution with CPP of the autoregulation indices BFAx and PRx, respectively. In columns 2, 3, and 4, the thick line represents the median value of $\mathrm{rCBF}_{\mathrm{i}}, \mathrm{BFAx}$, and $\mathrm{PRx}$, respectively, and the colored area shows the interquartile range.

\subsubsection{Case study: patient 1}

In patient 1 (Fig. 3), the $\mathrm{rCBF}_{\mathrm{i}}$ versus MAP curve shows a linear relationship with a slope of $2.5 \%$ flow increase per $\mathrm{mmHg}$ pressure increase. The high slope of the autoregulation curve $\mathrm{rCBF}_{\mathrm{i}}$ versus MAP suggests impaired autoregulation during this recording. ICP was high for this patient and difficult to manage. This would typically lead to lost autoregulation, in agreement with the $\mathrm{rCBF}_{\mathrm{i}}$ versus MAP curves.
The BFAx fluctuates between 0 and 0.5 over the $20-\mathrm{mmHg}$ range of CPP fluctuations. There is poor agreement between BFAx and the PRx index: PRx is high (around 0.5 for CPP above $60 \mathrm{mmHg}$ ), consistent with impaired autoregulation, whereas the fluctuating BFAx is difficult to interpret.

\subsubsection{Case study: patient 2}

The two recordings in patient 2 (Fig. 4) were obtained four days apart, with the probe located at a similar location, but the patient being in different clinical conditions. Notably, during the first session the patient showed generalized EEG activity, whereas the EEG activity had normalized during the second session. In the second session, the patient had undergone vasospasm therapy through vasopressor medication. It is, therefore, difficult to interpret the two recordings together. During the first recording, $\mathrm{rCBF}_{\mathrm{i}}$ presents three behaviors: at low MAP, it shows a strong increase with MAP (3\% per $\mathrm{mmHg}$ ); then, it remains relatively constant over the 85 to $105 \mathrm{mmHg}$ range; and finally, it increases sharply (almost $10 \%$ per $\mathrm{mmHg}$ ) at MAPs over $105 \mathrm{mmHg}$. The curve suggests a narrow plateau of regulated flow between 85 and $105 \mathrm{mmHg}$, with the lower and higher limit of autoregulation both being reached during that session.

During the second session, $\mathrm{rCBF}_{\mathrm{i}}$ is relatively constant over the 85 - to $120-\mathrm{mmHg}$ range of MAP values, and it
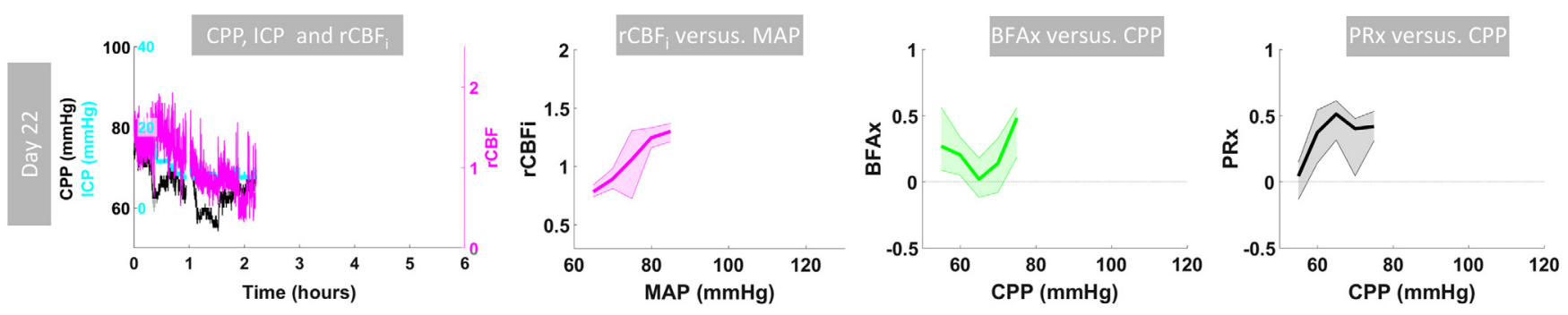

Fig. 3 Autoregulatory curves for patient 1 . The first panel displays the CPP, ICP, and $\mathrm{rCBF}_{\mathrm{i}}$ time traces over $6 \mathrm{~h}$ of recording. The second panel displays the $\mathrm{rCBF}_{\mathrm{i}}$ versus MAP curve for that recording. Panel 3 displays the association of the blood flow autoregulation index BFAx with CPP. Panel 4 displays the association of the reactivity index PRx with CPP. In panels 2, 3, and 4, the thick line represents the median value in that MAP (CPP) segment of $\mathrm{rCBF}_{\mathrm{i}}, \mathrm{BFAx}$, and PRx, respectively, and the colored area shows the interquartile range.
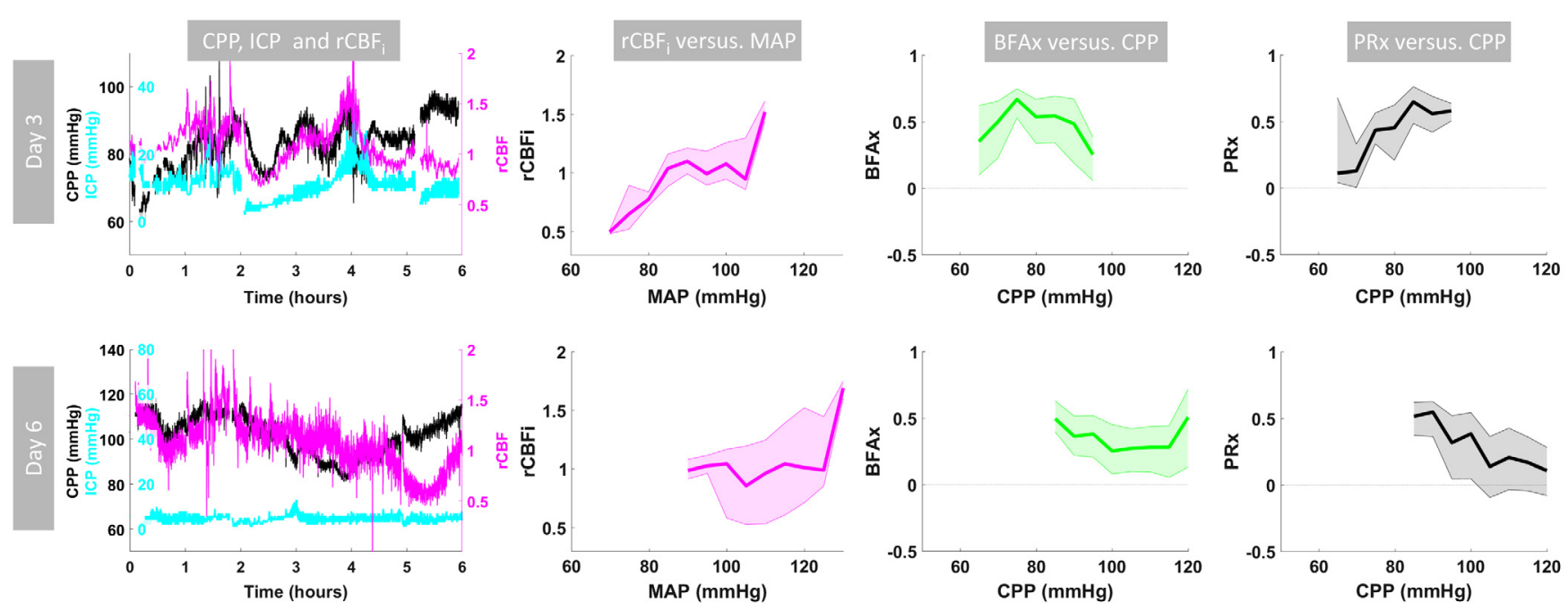

Fig. 4 Time series, autoregulatory curves, and autoregulation indices for patient 2. The two rows correspond to the 2 days of recordings. The panels are the same as for Fig. 3 . 

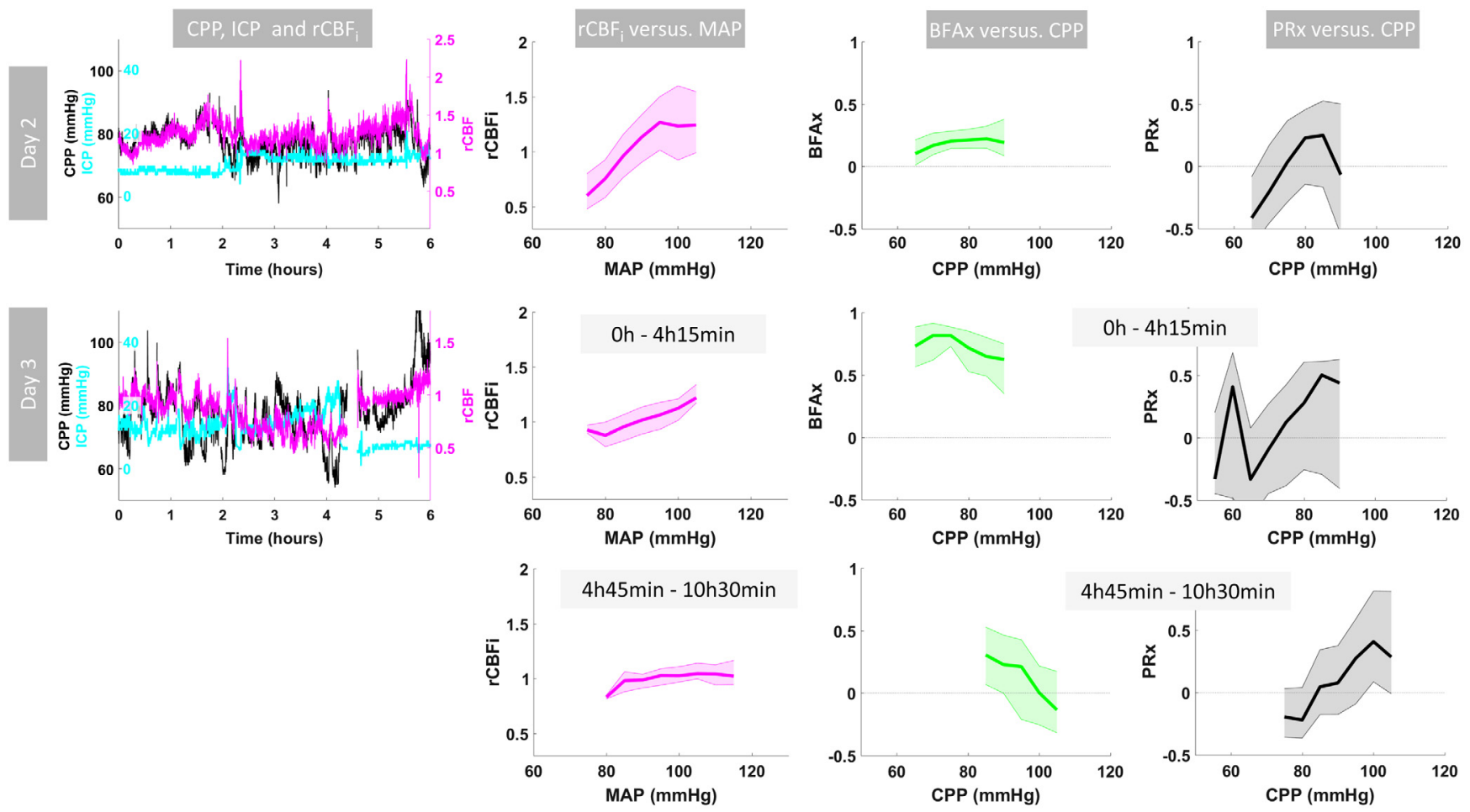

Fig. 5 Time series, autoregulatory curves, and autoregulation indices for patient 3 . The panels are the same as for Fig. 3.
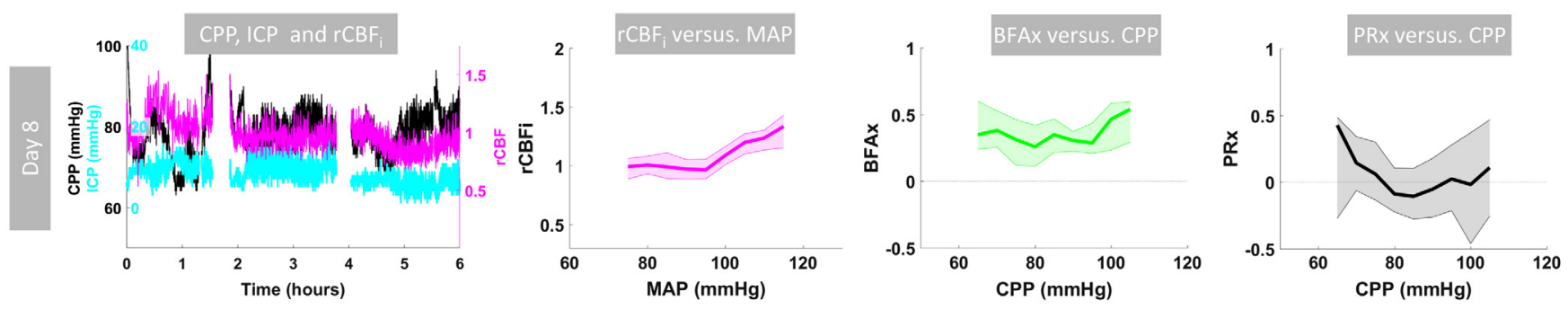

Fig. 6 Time series, autoregulatory curves, and autoregulation indices for patient 4 . The panels are the same as for Fig. 3.
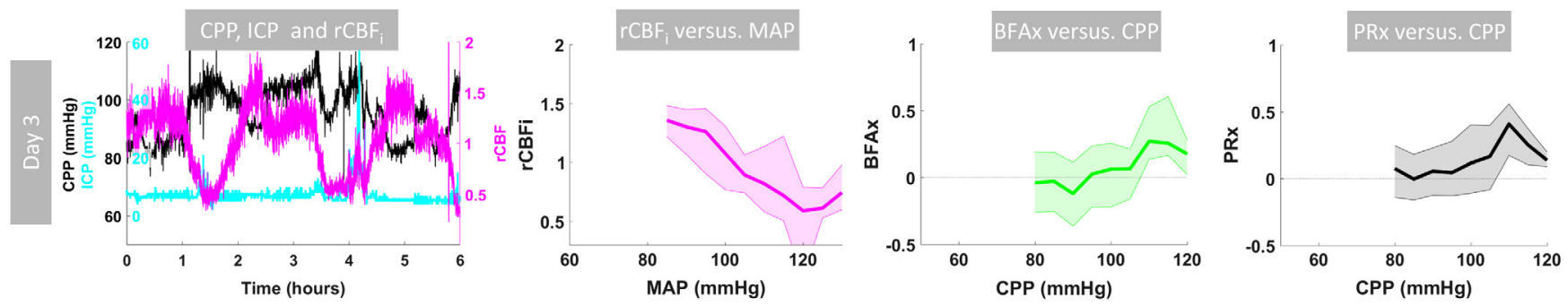

Fig. 7 Time series, autoregulatory curves, and autoregulation indices for patient 5 . The panels are the same as for Fig. 3.

increases sharply for MAP above $120 \mathrm{mmHg}$. The curve suggests improved CA on the second session, which would be consistent with the patient being in a less severe condition in general, after normalization of EEG activity and vasopressor therapy.

For both sessions, the BFAx and the PRx curves present some disparities but overall similar evolution and values, with both indices reaching very high values during the first session (up to 0.6 to 0.7 ) and slightly lower during the second session (up to 0.5). During the second session, both BFAx and PRx curves present a slight decrease with increasing CPP, showing good agreement with each other. This would, however, suggest better autoregulation at higher CPP, somewhat in contradiction with an increase in the CBFi at high MAP. 

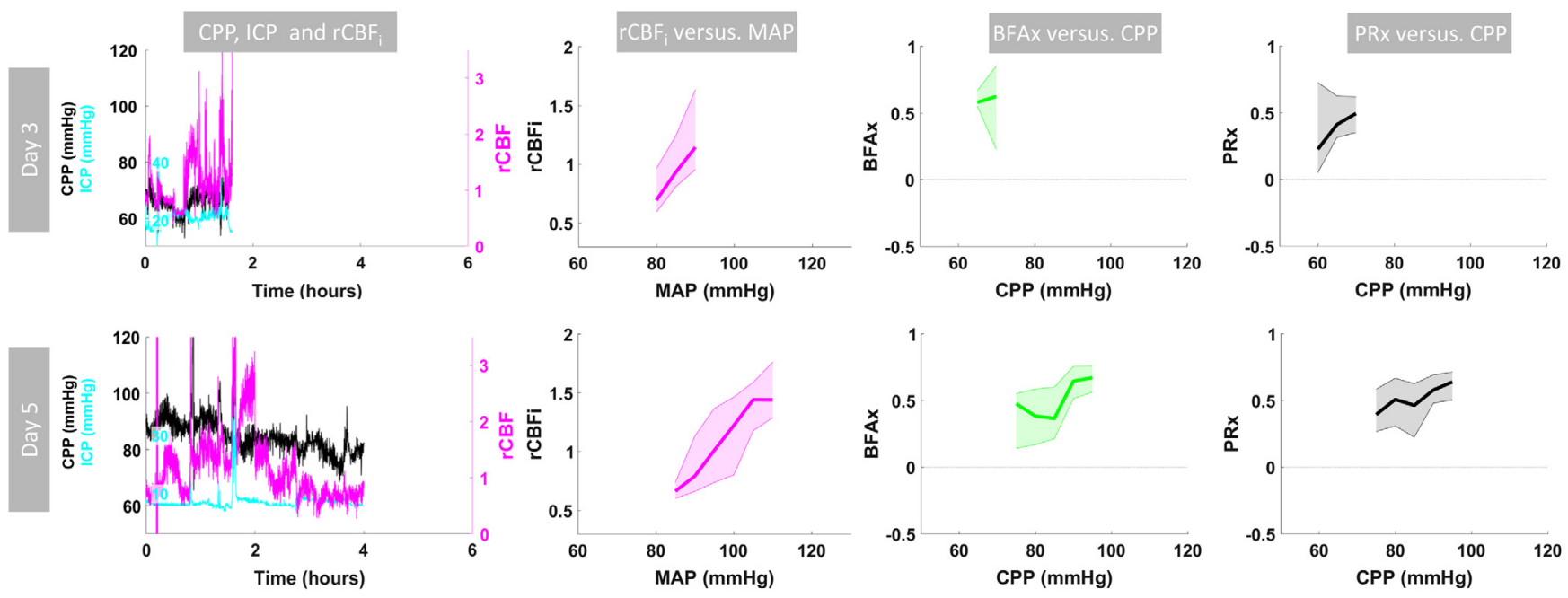

Fig. 8 Time series, autoregulatory curves, and autoregulation indices for patient 6 . The panels are the same as for Fig. 3 .

\subsubsection{Case study: patient 3}

Both recordings in patient 3 present similar trends (Fig. 5). The $\mathrm{rCBF}_{\mathrm{i}}$ and $\mathrm{CPP}$ temporal traces appear to be highly correlated, with $\mathrm{rCBF}_{\mathrm{i}}$ passively following all $\mathrm{CPP}$ fluctuations. This is confirmed by the autoregulation curve of the first session, showing a linear relationship between $\mathrm{rCBF}_{\mathrm{i}}$ and MAP, with a slope $>3 \% \mathrm{rCBF}_{\mathrm{i}}$ increase per $\mathrm{mmHg}$. During session 2, there was a sudden drop in ICP of $>10 \mathrm{mmHg}$ at about $t=4.5 \mathrm{~h}$ after the start of the recording, and we analyzed the segments before and after the drop separately. In the first segment, $\mathrm{rCBF}_{\mathrm{i}}$ varies linearly with MAP with a slop of about $2 \%$ per mmHg. In the second segment, after ICP has been reduced, the $\mathrm{rCBF}_{\mathrm{i}}$ versus MAP curve is almost constant with pressure, suggesting retrieved autoregulatory capacity.

The BFAx curves show high values for all recordings, especially the first segment of session 2, with BFAx remaining above 0.6 over the whole CPP range. In contrast, during the second segment, BFAx takes lower values, consistent with the interpretation of some retrieved autoregulation capacity after ICP has been decreased. There is, however, no agreement between the BFAx and the PRx curves for this patient, the latter showing highly fluctuating values with even negative values being reached. Note, however, that a dysfunction of the extra-ventricular drain for this patient complicated ICP management and monitoring.

In this patient, ICP was high during the two sessions reaching values of $20 \mathrm{mmHg}$ and above, even though it dropped below $10 \mathrm{mmHg}$ after $4.5 \mathrm{~h}$ of the second recording. This high ICP would typically impair the autoregulatory capacity of the brain, and likely explains the very high BFAx indices we observed. This patient's condition was severe, including low clinical measures of brain tissue oxygenation, which itself was blood pressure-dependent. This patient died during hospitalization.

\subsubsection{Case study: patient 4}

In patient 4 , the short separation DCS signal had low quality $\left(R^{2}\right.$ of the autocorrelation curve fit around 0.2), and we, therefore, could not use it to subtract it from the long separation signal. Instead, we simply present the result at the long separation
$\mathrm{BFi}_{\text {Long }}$ (Fig. 6). $\mathrm{rBFi}_{\text {Long }}$ is relatively constant over the 75 to $95 \mathrm{mmHg}$ range of MAP, then increases for MAP above $95 \mathrm{mmHg}$ (about $2 \%$ per $\mathrm{mmHg}$ ).

This interpretation is also consistent with the PRx index, which is low over the whole range of CPP fluctuations. However, the BFAx over the same range is relatively constant and high (0.3 to 0.4$)$.

\subsubsection{Case study: patient 5}

The results for patient 5 (Fig. 7) present a different trend than for the other patients, displaying an overall decrease of $\mathrm{rCBF}_{\mathrm{i}}$ with increasing MAP over the $85-$ to $125-\mathrm{mmHg}$ range. When looking at the temporal series, it appears that this inverse correlation is due to very slow CPP oscillations (30- to 60-min time scale) of large magnitude, inducing inverted response in $\mathrm{rCBF}_{\mathrm{i}}$. The behavior was the same when using $\mathrm{BFi}_{\text {Long }}$ instead of $\mathrm{CBF}_{\mathrm{i}}$ and therefore is not an artifact arising from the data processing. Of note, the patient was clinically noted during this time to demonstrate cycles of alternating hyperventilation and hypoventilation, and when examining the BFAx, which focuses on 10-s oscillations, we observe the expected behavior, with a low BFAx (around 0) for CPP in the 80- to $105-\mathrm{mmHg}$ range, which increases slightly to 0.2 for CPP above $110 \mathrm{mmHg}$. The reactivity index PRx presents the same behavior, with overall low values (around 0) and an increase for CPP values above $110 \mathrm{mmHg}$. We propose a few hypotheses for this paradoxical behavior of CBFi versus MAP in the discussion section below.

\subsubsection{Case study: patient 6}

In patient $6, \mathrm{rCBF}_{\mathrm{i}}$ increases sharply with MAP during the two recordings with a slope higher than $3 \%$ per $\mathrm{mmHg}$.

In agreement to the $\mathrm{rCBF}_{\mathrm{i}}$ linear dependency on MAP, BFAx appears very high (0.5) for both recordings. The PRx curves also support the same interpretation and are in good agreement with BFAx. These combined results suggest impaired autoregulation over the whole range of CPP.

During the first session, the patient presented high ICP (about $20 \mathrm{mmHg}$ ), which can explain the loss of autoregulation we observe from the CBFi and BFAx measures. The patient had improved on the second session and had lower ICP. 


\subsubsection{Overview of results}

There is a large variability in the observed autoregulatory behaviors across subjects and even across days for the same patient. This is expected for this heterogeneous population and in these very acute situations. We can, however, identify some common features across recordings and patients. Except for patient 5, there is a linear relationship between $\mathrm{CBF}_{\mathrm{i}}$ and MAP with a positive slope, suggestive of impaired autoregulation. Autoregulation appears to be more preserved around 90 to $110 \mathrm{mmHg}$ in most patients, as illustrated by a constant $\mathrm{CBF}_{\mathrm{i}}$ or a lower slope of the $\mathrm{CBF}_{\mathrm{i}}$ versus MAP relationship in that range. The exact range of MAP and the quality of observed regulation vary between patients. In all subjects, the results are consistent with those using $\mathrm{BFI}_{\text {Long }}$ (data not shown) instead of $\mathrm{CBFi}$, i.e., without subtracting a scalp component. The derived autoregulation index BFAx are compared with the PRx index reported in the literature. The behaviors of BFAx and PRx are only consistent in 5 out of 10 recordings.

\section{Discussion}

\subsection{Feasibility of Measurements}

The first goal of our study was to assess feasibility of long-term monitoring with DCS in neurocritical care patients. We could monitor patients with good signal quality for up to $16 \mathrm{~h}$ continuously. Measurements in this challenging population are complicated by multiple factors: heterogeneity of the pathology and severity across patients; presence of invasive and noninvasive neuromonitoring devices on the scalp limiting the space available for DCS measurements; deterioration of the DCS signal potentially arising from craniotomy, stitches, invasive bolt, edema, or extra-axial blood; and imaging exams or clinical care resulting in displacement of the probe. For these reasons, it was not possible in this pilot study to control for all parameters, and our measurement conditions present high variability across patients, such as day after admission, number and duration of recordings, location of the DCS probe, and exact sourcedetector separations.

Nonetheless, these first measurements allowed us to demonstrate the feasibility of DCS monitoring in the NeuroICU and to identify areas of improvements. For instance, we developed during the study a new probe, smaller, lighter, and more flexible, and therefore more adequate for this population.

\subsection{Interpretation of $\mathrm{rCBF}_{i}$ and Autoregulation Curves}

Due to the limitation of the multidistance frequency-domain method in the presence of a thick superficial layer and the constraints of space on the head, we did not attempt to measure the absolute optical properties of the head with our frequencydomain system. ${ }^{66-71}$ Instead, we assumed fixed optical properties and used a semi-infinite homogeneous model for the head anatomy. We, therefore, cannot trust the absolute values of $\mathrm{CBF}$ that we retrieve or compare them across subjects. For these reasons, we limited our analysis on an individual patient basis to relative blood flow changes that are independent from tissue optical properties.

The retrieved autoregulation curves that pass our quality criteria are physiologically plausible, showing linear dependency of flow versus pressure at low $(<90 \mathrm{mmHg}) \mathrm{MAP}$, and reaching a plateau of regulated flow at intermediate pressure (90 to $110 \mathrm{mmHg}$ ) in some patients.

The transition between the regions of preserved or impaired autoregulation occurs at a different perfusion pressure for each patient. This high variability in the results is not surprising considering the heterogeneity of the patients and measuring conditions.

Our data analysis relies on the assumption that autoregulation remains unchanged over the whole duration of each recording so that we can group all data points to derive a static autoregulation curve. However, it is possible that this assumption does not hold true, and that the autoregulatory capacity of each patient in this severely injured population varies dynamically over time. ${ }^{14}$ As future studies pair these data with clinical conditions or assess the impact of interventions, we note that the minimal duration necessary to achieve an autoregulatory will relate to how dynamic the range in $\mathrm{CBF}$ is; a restricted range of $\mathrm{CBF}$ will only minimally explore the autoregulatory capacity.

The second underlying assumption in interpreting our results is that we are monitoring the global autoregulatory capacity of the brain. In 4 out of 6 patients, the DCS probe was located on the hemisphere contralateral to the main injury, whereas in two patients, it was placed on the ipsilateral side. Available space around clinical neuromonitoring equipment on the scalp as well as DCS signal quality dictated these choices. As we are looking at a single location with a sensitivity limited to a few cubic centimeters below the probe, it is possible that multiregional or bihemispheric measurements would reveal regional heterogeneity in the autoregulation or metabolism of the brain, often a result of the heterogeneity of vasospasms, epileptiform and seizure activity, or cortical spreading depolarizations following SAH. ${ }^{72-75}$ Further studies monitoring different regions of the brain with additional DCS channels would be required to address this issue. Note, however, that, except for EEG, most neuromonitoring modalities only provide a global (ICP) or on the contrary a very local (laser Doppler flowmetry, brain tissue oxygen tension) surrogate of $\mathrm{CBF}$, or at best pauciregional measure (TCD, cerebral oximeter). We, therefore, believe that DCS has its space in the neuromonitoring arena to complement these other modalities, by providing a noninvasive, multiregional, and continuous measure of blood flow.

\subsection{Validation of $\mathrm{rCBF}_{i}$ and Autoregulation Curves with Other Modalities}

Despite the plausible interpretation of the autoregulation curves, the first main limitation of the present study at this stage is that we cannot validate these physiological interpretations as there is no standard measure of CBF or autoregulation in these patients. The closest measure of CBF that was available in two patients was white matter perfusion $\mathrm{BF}_{\mathrm{WM}}$ assessed with an invasive diffusion sensor. However, $\mathrm{BF}_{\mathrm{WM}}$ measurements are not always reliable as they are very sensitive to placement and require calibration. In one patient, $\mathrm{BF}_{\mathrm{WM}}$ and $\mathrm{rCBF}_{\mathrm{i}}$ were not correlated when considering the whole monitoring period. The two signals presented better correlation over shorter segments $(4 \mathrm{~h})$. It is possible that the low correlation over the whole recording arises from sudden offsets in the signals or from a significant clinical change midrecording. In addition, the two devices measure two different parameters often in different locations. With these caveats in mind, the observed significant correlation between the invasive measure of perfusion and the DCS-derived $\mathrm{CBF}_{\mathrm{i}}$, on 
patient 3 during the whole recordings, and in patient 2 over a few hours, provides some confidence in the noninvasive measure of CBF we obtain with DCS.

\subsection{Extra-Cerebral Contamination}

The second major limitation of our study is the unknown magnitude of the extra-cerebral contribution to the DCS signal. The issue is particularly challenging in the NeuroICU population as all patients were severely injured and therefore susceptible to strongly impaired CA. It complicates the interpretation of the lack of autoregulation we observed in some patients: it could arise from partial contamination of the signal by scalp flow, which is not regulated, or it could be a true measure of severely disrupted autoregulation in the brain.

Different methodologies have been proposed to assess the contribution from extracerebral flow, including measurements at different pressures to modulate scalp flow, ${ }^{44,76}$ and/or multilayer modeling of the head. ${ }^{77}$ Here, with the limited information available, we opted for a simple estimate of $\mathrm{CBF}$, obtained by subtracting a fixed portion $(70 \%)$ of the $\mathrm{BF}_{\mathrm{i}}$ value at the short separation from that at the long separation. This is a rough approximation, and the method is likely to over- or under-estimate the scalp contribution depending on individual patient's anatomy and source-detector separation. Further measurements and modeling studies will be required to improve the quantification of CBF and the minimization of extra-cerebral flow.

In parallel, different paths are conceivable in the future to improve brain sensitivity during clinical monitoring: increased source-detector separation with higher source power to maintain reasonable signal-to-noise ratio; optimization of the DCS probe location on an individual basis after observation of the patient's CT or MRI scan to minimize the distance to the brain; implementation of time-domain DCS, a recent technological innovation with improved depth sensitivity. ${ }^{39}$

\subsection{Consistency Between $\mathrm{rCBF}_{i}$ and BFAx}

We introduced the BFAx index of autoregulation to characterize the correlation between MAP and CBFi fluctuations. It is modeled on other indices defined in the literature, which have shown utility for monitoring CA in SAH patients. For instance, Soehle et al. $^{78}$ showed increased $\mathrm{Mx}$ during cerebral vasospasm, demonstrating impaired CA. Jaeger et al. ${ }^{79}$ observed that ORx, an index of brain tissue oxygen pressure reactivity, indicated impaired autoregulation in patients who develop delayed infarction after SAH.

In general, the observed results for the $\mathrm{rCBF}_{\mathrm{i}}$ curves and the BFAx curves are consistent, in that a high slope of $\mathrm{rCBF}_{\mathrm{i}}$ versus MAP is accompanied by a high reactivity index, illustrative of disrupted autoregulation, whereas regions of mainly constant $\mathrm{rCBF}_{\mathrm{i}}$ correspond to low BFAx, suggestive of preserved autoregulation. There are a few exceptions though, notably in patient 5 , who exhibits an inverted response in CBF that decreases for increasing CPP while the BFAx curves presents the expected behavior. As mentioned before, it is interesting to note that the inverted response arises from very slow fluctuations (30 to $60 \mathrm{~min}$ ) while the value of the reactivity index arises from faster LFOs $(10 \mathrm{~s})$. The reason for the paradoxical response in $\mathrm{rCBF}_{\mathrm{i}}$ at very low frequencies is unclear, but the clinical association of this finding was with a cyclic variation in respiratory rate (recurring cycles of hyperventilation followed by hypoventilation), in which the hyperventilatory portion of the cycle is typically associated with increased arousal (Cheyne-Stoke respiratory pattern) and thus may increase MAP while the hyperventilation would directly lower $\mathrm{CBF}$. In general, a continuous index of autoregulation based on $10 \mathrm{~s}$ time-scale oscillations may be more appropriate than the static curve of autoregulation to describe the fluctuations of autoregulatory capacity in these patients.

While both the reactivity index PRx and this newly introduced autoregulation index BFAx should reflect the autoregulatory capacity of the brain, the agreement between the two is only modest in the present study, with only 5 recordings out of 10 displaying consistent behaviors between the two indices. The disagreement between the two indices could arise from a number of reasons. First, it is important to note that they reflect two different cerebral metrics: ICP, which is believed to reflect $\mathrm{CBV}$, and the DCS-derived measure of CBF. Second, while $\mathrm{PRx}$ assesses a global vasomotor reactivity, ${ }^{80}$ BFAx reflects a regional vascular autoregulation. Previous studies have noted discrepancies between PRx and the mean index of cerebrovascular autoregulation $\mathrm{Mx}$, derived from TCD measure of CBFv. ${ }^{15,81}$ In a cohort of 345 patients, ${ }^{81}$ there was only moderate agreement between PRx and Mx, and the difference between the two indices was stronger for high values of ICP $(30 \mathrm{mmHg})$. One explanation proposed by Zweifel et al. ${ }^{15}$ is that the brain compliance at low ICP levels could buffer CBV changes, resulting in a low PRx, whereas for ICP rising above $30 \mathrm{mmHg}$, PRx increases sharply. While ICP was generally high in the patients we measured, it rarely rose above $20 \mathrm{mmHg}$. In addition, Zweifel et al. noted that despite the differences between PRx and $\mathrm{Mx}$, the two indices nonetheless showed a highly significant correlation of 0.4 to 0.6 . We observe larger differences in our data.

In addition to the fact that PRx and BFAx derive from distinct physiological quantities (CBV or vessel diameter, versus $\mathrm{CBF}$ ), and reflect processes of different spatial extents (global versus regional), other factors can explain the discrepancy between the two indices. For instance, potential delays between CPP and ICP or $\mathrm{CBF}_{\mathrm{i}}$ fluctuations will not be captured by the zero-lag correlation indices. Finally, the measure of ICP can sometimes be unreliable due to the difficulty of ventricular drain placement, as observed for instance in patient 3 .

Because of these limitations, the BFAx index will require further investigation in larger studies, ideally with a simultaneous invasive measure of cerebral perfusion for validation. It may provide a future avenue for monitoring autoregulation both continuously and noninvasively in patients with less severe conditions.

\section{Conclusion}

In this study, our main goal was to demonstrate the feasibility of long-term monitoring of CBF and autoregulation with DCS in high-risk NeuroICU patients. We acquired quality data continuously for up to $16 \mathrm{~h}$ in each patient. The DCS measure of blood flow index was recorded synchronously with MAP clinical monitoring, allowing us to derive autoregulation curves and to compute an autoregulation index BFAx. In general, our data suggest that the monitored patients had severely disrupted CA. Further measurements and data modeling are required to improve our physiological interpretation and to validate our conclusions.

To our knowledge, this is the first study demonstrating longterm monitoring of SAH patients with DCS. The modality offers 
a promising opportunity for continuous, noninvasive, regional monitoring of CBF in neurocritical care population, with the potential to be extended to multilocation measurements. The noninvasive measure of $\mathrm{CBF}$ would allow ubiquitous monitoring of patients with a range of injury severity, and down-the-road could enable subject-specific pressure management strategies to directly optimize brain perfusion rather than surrogate metrics.

\section{Disclosures}

MAF and DAB have financial interest in 149 Medical, Inc., a company developing DCS technology for assessing and monitoring cerebral blood flow in newborn infants, and in Dynometrics, Inc., a company that makes devices that use NIRS technology for athletes to evaluate muscle performance. MAF and DAB interests were reviewed and are managed by Massachusetts General Hospital and Partners HealthCare in accordance with their conflict of interest policies.

\section{Acknowledgments}

The authors are grateful to Zachary Starkweather, Davide Tamborini, and Bernhard Zimmermann for help with the DCS instrumentation and probes, and to Siddharth Biswal for assistance in extracting the clinical data. This work was supported by the National Institutes of Health Grant Nos. NINDS R21-NS094828, NIGMS R01-GM116177, 1S10RR023401, 1S10RR019307, and 1S10RR023043, the Andrew David Heitman Foundation, and by the Massachusetts General Hospital Executive Committee on Research (ECOR).

\section{References}

1. R. B. Panerai, "Cerebral autoregulation: from models to clinical applications," Cardiovasc. Eng. 8(1), 42-59 (2008).

2. C. Werner and K. Engelhard, "Pathophysiology of traumatic brain injury," Br. J. Anaesth. 99(1), 4-9 (2007).

3. W. J. Powers et al., "Autoregulation of cerebral blood flow surrounding acute (6 to 22 hours) intracerebral hemorrhage," Neurology 57(1), 18-24 (2001).

4. M. Czosnyka et al., "Monitoring of cerebral autoregulation in headinjured patients," Stroke 27, 1829-1834 (1996).

5. M. Czosnyka et al., "Cerebral autoregulation following head injury," J. Neurosurg. 95(5), 756-763 (2001).

6. L. A. Steiner et al., "Continuous monitoring of cerebrovascular pressure reactivity allows determination of optimal cerebral perfusion pressure in patients with traumatic brain injury," Crit. Care Med. 30(4), 733-738 (2002).

7. M. Reinhard et al., "Dynamic cerebral autoregulation in acute ischemic stroke assessed from spontaneous blood pressure fluctuations," Stroke 36(8), 1684-1689 (2005).

8. A. Dagal and A. M. Lam, "Cerebral blood flow and the injured brain: how should we monitor and manipulate it?" Curr. Opin. Anaesthesiol. 24(2), 131-137 (2011).

9. R. S. Frackowiak et al., "Quantitative measurement of regional cerebral blood flow and oxygen metabolism in man using $15 \mathrm{O}$ and positron emission tomography: theory, procedure, and normal values," $J$. Comput. Assist. Tomogr. 4(6), 727-736 (1980).

10. E. L. Barbier, L. Lamalle, and M. Décorps, "Methodology of brain perfusion imaging," J. Magn. Reson. Imaging 13(4), 496-520 (2001).

11. J. M. Lam, J. N. Hsiang, and W. S. Poon, "Monitoring of autoregulation using laser Doppler flowmetry in patients with head injury," $J$. Neurosurg. 86(3), 438-445 (1997).

12. P. Vajkoczy et al., "Continuous monitoring of regional cerebral blood flow: experimental and clinical validation of a novel thermal diffusion microprobe," J. Neurosurg. 93(2), 265-274 (2000).

13. W. A. van den Brink et al., "Brain oxygen tension in severe head injury," Neurosurgery 46(4), 868-876 (2000).
14. M. Czosnyka et al., "Monitoring of cerebrovascular autoregulation: facts, myths, and missing links," Neurocrit. Care 10(3), 373-386 (2009).

15. C. Zweifel et al., "Continuous time-domain monitoring of cerebral autoregulation in neurocritical care," Med. Eng. Phys. 36(5), 638-645 (2014).

16. N. L. Bishop et al., "Transcranial doppler measurement of middle cerebral artery blood flow velocity: a validation study," Stroke 17(5), 913-915 (1986).

17. R. B. Panerai, "Transcranial doppler for evaluation of cerebral autoregulation," Clin. Auton. Res. 19(4), 197-211 (2009).

18. M. Ono et al., "Validation of a stand-alone near-infrared spectroscopy system for monitoring cerebral autoregulation during cardiac surgery," Anesth. Analg. 116(1), 198-204 (2013).

19. L. A. Steiner et al., "Near-infrared spectroscopy can monitor dynamic cerebral autoregulation in adults," Neurocrit. Care 10(1), 122-128 (2009).

20. K. M. Brady et al., "Monitoring cerebral blood flow pressure autoregulation in pediatric patients during cardiac surgery," Stroke 41(9), 19571962 (2010).

21. K. Brady et al., "Real-time continuous monitoring of cerebral blood flow autoregulation using near-infrared spectroscopy in patients undergoing cardiopulmonary bypass," Stroke 41(9), 1951-1956 (2010).

22. D. A. Boas and M. A. Franceschini, "Haemoglobin oxygen saturation as a biomarker: the problem and a solution," Philos. Trans. A. Math. Phys. Eng. Sci. 369(1955), 4407-4424 (2011).

23. M. Reinhard et al., "Oscillatory cerebral hemodynamics-the macrovs. microvascular level." J. Neurol. Sci. 250(1-2), 103-109 (2006).

24. S. Muehlschlegel et al., "Feasibility of NIRS in the neurointensive care unit: a pilot study in stroke using physiological oscillations," Neurocrit. Care 11(2), 288-295 (2009).

25. M. Reinhard et al., "Spatial mapping of dynamic cerebral autoregulation by multichannel near-infrared spectroscopy in high-grade carotid artery disease," J. Biomed. Opt. 19(9), 097005 (2014).

26. J. M. Kainerstorfer et al., "Cerebral autoregulation in the microvasculature measured with near-infrared spectroscopy," J. Cereb. Blood Flow Metab. 35(6), 959-966 (2015).

27. S. J. Payne, J. Selb, and D. A. Boas, "Effects of autoregulation and $\mathrm{CO}_{2}$ reactivity on cerebral oxygen transport," Ann. Biomed. Eng. 37(11), 2288-2298 (2009).

28. R. Saager and A. Berger, "Measurement of layer-like hemodynamic trends in scalp and cortex: implications for physiological baseline suppression in functional near-infrared spectroscopy," J. Biomed. Opt. 13(3), 034017 (2008).

29. S. Gunadi et al., "Spatial sensitivity and penetration depth of three cerebral oxygenation monitors," Biomed. Opt. Express 5(9), 2896 (2014).

30. Q. Zhang, E. N. Brown, and G. E. Strangman, "Adaptive filtering to reduce global interference in evoked brain activity detection: a human subject case study," J. Biomed. Opt. 12(6), 064009 (2007).

31. L. Gagnon et al., "Further improvement in reducing superficial contamination in NIRS using double short separation measurements," Neuroimage 85(Pt 1), 127-135 (2014).

32. N. Roche-Labarbe et al., "Noninvasive optical measures of CBV, $\mathrm{StO}(2)$, CBF Index, and $\mathrm{rCMRO}(2)$ in human premature neonates' brains in the first six weeks of life," Hum. Brain Mapp. 31(3), 341352 (2010).

33. M. N. Kim et al., "Noninvasive measurement of cerebral blood flow and blood oxygenation using near-infrared and diffuse correlation spectroscopies in critically brain-injured adults," Neurocrit. Care 12(2), 173-180 (2010).

34. S. A. Carp et al., "Validation of diffuse correlation spectroscopy measurements of rodent cerebral blood flow with simultaneous arterial spin labeling MRI; towards MRI-optical continuous cerebral metabolic monitoring," Biomed. Opt. Express 1, 553-565 (2010).

35. E. M. Buckley et al., "Validation of diffuse correlation spectroscopic measurement of cerebral blood flow using phase-encoded velocity mapping magnetic resonance imaging," J. Biomed. Opt. 17(3), 037007 (2012).

36. J. Selb et al., "Sensitivity of near-infrared spectroscopy and diffuse correlation spectroscopy to brain hemodynamics: simulations and experimental findings during hypercapnia," Neurophotonics 1(1), 015005 (2014).

37. D. A. Boas and A. G. Yodh, "Spatially varying dynamical properties of turbid media probed with diffusing temporal light correlation," J. Opt. Soc. Am. A 14(1), 192-215 (1997). 
38. D. Wang et al., "Fast blood flow monitoring in deep tissues with real-time software correlators," Biomed. Opt. Express 7(3), 776 (2016).

39. J. Sutin et al., "Time-domain diffuse correlation spectroscopy," Optica 3(9), 1006-1013 (2016)

40. D. A. Boas "Establishing the relationship of diffuse correlation spectroscopy signal with blood flow," Neurophotonics 3(3), 031412 (2016).

41. S. A. Carp et al., "Due to intravascular multiple sequential scattering, diffuse correlation spectroscopy of tissue primarily measures relative red blood cell motion within vessels," Biomed. Opt. Express 2(7), 2047-2054 (2011).

42. W. B. Baker et al., "Modified Beer-Lambert law for blood flow," Biomed. Opt. Express 5(11), 4053 (2014).

43. P. Farzam and T. Durduran, "Multi-distance diffuse correlation spectroscopy for simultaneous estimation of absolute scattering and absorption coefficient and the blood flow index," J. Biomed. Opt. 20, 055001 (2015).

44. W. Baker et al., "Probe pressure modulation algorithm reduces extracerebral contamination in optical measurements of cerebral blood flow," in Biomedical Optics, p. BS3A.52 (2014).

45. L. X. Dong et al., "Simultaneously extracting multiple parameters via fitting one single autocorrelation function curve in diffuse correlation spectroscopy," IEEE Trans. Biomed. Eng. 60(2), 361-368 (2013).

46. T. Durduran et al., "Transcranial optical monitoring of cerebrovascular hemodynamics in acute stroke patients," Opt. Express 17(5), 3884 3902 (2009).

47. M. N. Kim et al., "Continuous optical monitoring of cerebral hemodynamics during head-of-bed manipulation in brain-injured adults," Neurocrit. Care 20(3), 443-453 (2014).

48. P. Zirak et al., "Transcranial diffuse optical monitoring of microvascular cerebral hemodynamics after thrombolysis in ischemic stroke," J. Biomed. Opt. 19(1), 018002 (2014).

49. P. Zirak et al., "Microvascular versus macrovascular cerebral vasomotor reactivity in patients with severe internal carotid artery stenosis or occlusion," Acad. Radiol. 21(2), 168-174 (2014).

50. E. M. Buckley et al., "Early postoperative changes in cerebral oxygen metabolism following neonatal cardiac surgery: effects of surgical duration," J. Thorac. Cardiovasc. Surg. 145, 196-205 (2013).

51. M. Dehaes et al., "Perioperative cerebral hemodynamics and oxygen metabolism in neonates with single-ventricle physiology," Biomed. Opt. Express 6(12), 4749 (2015).

52. Y. Shang et al., "Cerebral monitoring during carotid endarterectomy using near-infrared diffuse optical spectroscopies and electroencephalogram," Phys. Med. Biol. 56, 3015-3032 (2011).

53. D. R. Busch et al., "Continuous cerebral hemodynamic measurement during deep hypothermic circulatory arrest," Biomed. Opt. Express 7(9), 3461-3470 (2016).

54. S. L. Ferradal et al., "Non-invasive assessment of cerebral blood flow and oxygen metabolism in neonates during hypothermic cardiopulmonary bypass: feasibility and clinical implications," Sci. Rep. 7(March), 44117 (2017).

55. R. Cheng et al., "Noninvasive optical evaluation of spontaneous low frequency oscillations in cerebral hemodynamics," Neuroimage 62, 1445-1454 (2012).

56. P.-Y. Lin et al., "Non-invasive optical measurement of cerebral metabolism and hemodynamics in infants," J. Vision Exp. 14(73), e4379 (2013).

57. T. Durduran et al., "Optical measurement of cerebral hemodynamics and oxygen metabolism in neonates with congenital heart defects," J. Biomed. Opt. 15(3), 037004 (2010).

58. M. Dehaes et al., "Cerebral oxygen metabolism in neonatal hypoxic ischemic encephalopathy during and after therapeutic hypothermia," J. Cereb. Blood Flow Metab. 34(1), 87-94 (2014).

59. E. M. Buckley et al., "Cerebral hemodynamics in preterm infants during positional intervention measured with diffuse correlation spectroscopy and transcranial Doppler ultrasound," Opt. Express 17, 12571-12581 (2009).

60. N. A. Lassen, "Cerebral blood flow and oxygen consumption in man," Physiol. Rev. 39(2), 183-238 (1959).

61. T. Durduran et al., "Diffuse optical measurement of blood flow, blood oxygenation, and metabolism in a human brain during sensorimotor cortex activation," Opt. Lett. 29(15), 1766-1768 (2004).

62. D. Tamborini et al., "Development and characterization of a multidistance and multiwavelength diffuse correlation spectroscopy system," Neurophotonics 5(1), 011015 (2017).
63. J. Sutin, P. Y. Lin, and M. A. Franceschini, "System and method for improved light delivery to and from subjects," U. S. Patent Application No. 20170027447A1.

64. Laser Institute of America, "American National Standard for Safe Use of Lasers," no. 56825 (2011).

65. E. Ohmae et al., "Cerebral hemodynamics evaluation by near-infrared time-resolved spectroscopy: correlation with simultaneous positron emission tomography measurements," Neuroimage 29, 697-705 (2006).

66. S. Fantini et al., "Frequency-domain multichannel optical-detector for noninvasive tissue spectroscopy and oximetry," Opt. Eng. 34, 32-42 (1995).

67. M. A. Franceschini et al., "Assessment of infant brain development with frequency-domain near-infrared spectroscopy," Pediatr. Res. 61(5), 546-551 (2007).

68. B. Hallacoglu, A. Sassaroli, and S. Fantini, "Optical characterization of two-layered turbid media for non-invasive, absolute oximetry in cerebral and extracerebral tissue," PLoS One 8(5), e64095 (2013).

69. M. S. Patterson, B. Chance, and B. C. Wilson, "Time resolved reflectance and transmittance for the non-invasive measurement of tissue optical properties," Appl. Opt. 28(12), 2331-2336 (1989).

70. F. Martelli et al., "Solution of the time-dependent diffusion equation for layered diffusive media by the eigenfunction method," Phys. Rev. E 67(5), 1-14 (2003).

71. J. Selb et al., "Comparison of a layered slab and an atlas head model for Monte Carlo fitting of time-domain near-infrared spectroscopy data of the adult head," J. Biomed. Opt. 19(1), 016010 (2014).

72. E. S. Connolly et al., "Guidelines for the management of aneurysmal subarachnoid hemorrhage: a guideline for healthcare professionals from the American Heart Association/American Stroke Association," Stroke 43(6), 1711-1737 (2012).

73. G. V Varsos et al., "Model-based indices describing cerebrovascular dynamics," Neurocrit. Care 20, 142-157 (2014).

74. J. A. Hartings et al., "Spreading depression in continuous electroencephalography of brain trauma," Ann. Neurol. 76(5), 681-694 (2014).

75. A. F. Struck et al., "Metabolic correlates of the Ictal-Interictal Continuum: FDG-PET during continuous EEG," Neurocrit. Care 24(3), 324-331 (2016).

76. R. C. Mesquita et al., "Influence of probe pressure on the diffuse correlation spectroscopy blood flow signal: extra-cerebral contributions," Biomed. Opt. Express 4(7), 978-994 (2013).

77. L. Gagnon et al., "Investigation of diffuse correlation spectroscopy in multi-layered media including the human head," Opt. Express 16(20), 15514-15530 (2008).

78. M. Soehle et al., "Continuous assessment of cerebral autoregulation in subarachnoid hemorrhage," Anesth. Analg. 98(4), 1133-1139 (2004).

79. M. Jaeger et al., "Continuous monitoring of cerebrovascular autoregulation after subarachnoid hemorrhage by brain tissue oxygen pressure reactivity and its relation to delayed cerebral infarction," Stroke 38(3), 981-986 (2007)

80. C. Zweifel et al., "Continuous monitoring of cerebrovascular pressure reactivity in patients with head injury," Neurosurg. Focus 25(4), E2 (2008).

81. K. Budohoski et al., "the relationship between cerebral blood flow autoregulation and cerebrovascular pressure reactivity after traumatic brain injury," Neurosurgery 71(3), 652-661 (2012).

Juliette Selb is a senior research scientist at Boston University and former instructor at the Optics at Martinos, Massachusetts General Hospital, Harvard Medical School. She received her PhD in 2002 from the Université Paris Sud in France for her work on acousto-optic imaging. Her current research focuses on diffuse optical modalities for brain imaging in humans.

Kuan-Cheng $\mathbf{W u}$ is a graduate student at Boston University and a research assistant at optics at Martinos, Massachusetts General Hospital, Harvard Medical School.

Jason Sutin is a postdoc at Boston Children's Hospital and former research assistant at the optics at Martinos, Massachusetts General Hospital, Harvard Medical School.

Pei-Yi (Ivy) Lin is an assistant professor at Boston Children's Hospital and former instructor at the optics at Martinos, Massachusetts General Hospital, Harvard Medical School. 
Parisa Farzam is a research fellow at the Optics at Martinos, Massachusetts General Hospital, Harvard Medical School. She received her $\mathrm{PhD}$ in biomedical photonics from ICFO-The Institute of Photonics Sciences, Spain. Her research is focused on developing novel diffuse optical instrumentation, algorithms and protocols and applying them for preclinical and clinical investigations.

Sophia Bechek is a medical student and former clinical research coordinator in the MGH NeurolCU.

Apeksha Shenoy is a clinical research coordinator in the MGH NeurolCU.

Aman B. Patel is the director of open and endovascular neurosurgery at Massachusetts General Hospital, specializing in the treatment of vascular disorders of the brain and spine, including cerebral aneurysms, vascular malformations, and stroke.

David A. Boas is the director of the Neurophotonics Center at Boston University and a professor of Biomedical Engineering at Boston University. He received his BS degree in physics at Rensselaer Polytechnic Institute and his $\mathrm{PhD}$ in physics at the University of Pennsylvania. He is the founding president of the Society for
Functional Near-Infrared Spectroscopy, founding editor-in-chief of Neurophotonics, and the recipient of the 2016 Britton Chance Award in biomedical optics.

Maria Angela Franceschini is an associate professor at Harvard Medical School with specific training and expertise in the development of noninvasive optical techniques and applications in neuroscience, neurology, and brain health. As a pioneer in the field of near-infrared spectroscopy (NIRS), she has made substantial contributions to the development of NIRS instruments and to the modeling and testing of diffusion theory to describe light propagation in turbid media. She has successfully applied the technology to a large range of functional neuroimaging and clinical neuromonitoring applications.

Eric S. Rosenthal is an assistant professor at Harvard Medical School and the medical director of the MGH Critical Care Neurology Service, where he works as a neurointensivist and a clinical neurophysiologist. His academic focus is the use of multiparameter physiologic monitoring, time-series analysis, electronic health record informatics, and data integration to characterize the pathophysiologic transitions to secondary neuronal injury as a means to developing targeted therapies that improve clinical outcomes following acute brain injury. 